\title{
HELMINTOSES INTESTINAIS \\ II - PREVALÊNCIA E CORRELAÇÃO COM RENDA, TAMANHO DA FAMÍLIA, ANEMIA E ESTADO NUTRICIONAL*
}

\author{
Elisete Silva Pedrazzani** \\ Dalva A. Mello** \\ Sergio Pripas*** \\ Marta Fucci*** \\ Calógeras A. de A. Barbosa** \\ Maria Cristina M. Santoro***
}

\begin{abstract}
PEDRAZZANI, E.S. et al. Helmintoses intestinais. II - - Prevalência e correlação com renda, tamanho da família, anemia e estado nutricional. Rev. Saúde públ., S. Paulo, 22:384-9, 1988.

RESUMO: Foi analisada a prevalência das helmintoses intestinais e suas associaçðes com anemia, estado nutricional, renda e composição familiar em escolares de Santa Eudoxia, subdistrito de São Carlos, SP, Brasil. Foram examinadas 254 crianças: dos resultados coprológicos, $37,8 \%$ foram positivos. O parasita mais frequente foi o Ascaris lumbricoides $(12,1 \%)$, seguido de Trichuris trichiura $(4,8 \%)$, ancilostomideo $(3,7 \%)$ e Hymenolepis nana $(3,7 \%)$. Ocorreu maior concentração de resultados positivos na faixa de 8 a 12 anos $(51,9 \%)$. Foram diagnosticadas como anêmicas $14,7 \%$ das crianças e $15 \%$ como desnutridas. Das correlaçóes estudadas, apenas aquela entre helmintose e tamanho familiar mostrou resultado estatisticamente significante.
\end{abstract}

UNITERMOS: Helmintiase, incidência. Inquéritoș epidemiológicos. Anemia, incidência. Estado nutricional. Renda. Composiçảo familiar. Fezes, análise.

\section{INTRODUÇÃO}

As helmintoses intestinais, ao lado da inadequada ingestão de alimentos, têm sido consideradas, por alguns autores $8,11,12$ como fatores primordiais na fisiopatologia da anemia e da desnutrição protéico-calórica. Debilitando a população e incapacitando o indivíduo para o bom desempenho de suas atividades físicas e intelectuais, as helmintoses constituem ainda um sério problema de saúde pública em nosso meio (Mello e col. ${ }^{6}, 1987$ ).

Todavia, essa convicção, embora fortemente estabelecida no passado, é na atualidade objeto de cuidadosa revisão por parte de diversos autores que investigam o significado da ação dos parasitas na gênese das distrofias 1,2,3,5,12.

O presente trabalho que faz parte de um projeto de pesquisa sobre intervenção em helmintoses intestinais em Santa Eudóxia (Município de São Carlos, Estado de São Paulo), tem como objetivo central a determinação da prevalência das verminoses nesse subdistrito e a avaliação de suas relaçōes com anemia, estado nutricional, renda e composição familiar, fornecendo subsídios para o desenvolvimento de açðes concretas a serem realizadas junto à população.

\section{MATERIAL E MÉTODOS}

A amostra analisada compreendeu 254 escolares matriculados na Escola Parque Municipal e na Escola Estadual do Primeiro Grau, no subdistrito de Santa Eudóxia, Município de São Carlos (Estado de São Paulo), entre os quais 205 moravam na Vila e 49 na zona de lavoura. Esse total correspondeu a $30 \%$ das crianças em idade escolar residentes no subdistrito.

Para coleta de informações foram elaboradas duas fichas: uma com dados que objetivavam traçar o perfil sócio-econômico da população (composição familiar, renda e condições habitacionais) e outra com a finalidade de levantar dados de cada criança (identificação, antropometria e análises laboratoriais).

\footnotetext{
* Subvencionado pelo Fomento de Educação Sanitária e Imunização em Massa contra Doenças Transmissiveis (FESIMA) da Secretaria de Estado da Saúde de São Paulo e Conselho Nacional de Desenvolvimento Científico e Tecnológico (CNPq) - Processo n? 406472/84-MP. Apresentado ao X Congresso da Sociedade Brasileira de Parasitologia. Salvador, Bahia, 1987.

* Centro de Ciências Biológicas e da Saúde (CCBS) da Universidade Federal de São Carlos. Caixa Postal 676 - 13560 São Carlos, SP - Brasil.

*** Centro de Saúde I - São Carlos - Rua Amadeu Amaral, s/n? - 13560 - São Carlos, SP - Brasil.
} 
O peso foi obtido usando-se balança Filizola calibrada diariamente, provida de braço metálico para aferir a estatura. O diagnóstico de desnutrição foi feito com base no gráfico de altura e peso do estudo antropométrico de Santo André ${ }^{4}$, sendo considerada desnutrida a criança com peso abaixo do percentil 10 .

A partir de uma amostra de $5 \mathrm{ml}$ de sangue venoso em EDTA foi feita a determinação da hemoglobina pelo método da cianometahemoglobina ${ }^{7}$. Para o diagnóstico de anemia foi utilizado o critério da $\mathrm{OMS}^{10}$ que considera $11 \mathrm{~g} / \mathrm{de}$ hemoglobina como limite inferior da normalidade, nesse grupo populacional.

Foi empregado o método de sedimentação de Lutz nos exames coprológicos. Nos casos em que os primeiros resultados foram negativos, os exames foram repetidos. O primeiro levantamento coprológico foi realizado no início do segundo semestre letivo de 1985; o segundo e o terceiro no início de cada semestre de 1986 . Foi feito um acompanhamento das crianças que participaram dos três levantamentos consecutivos, para se verificar o perfil evolutivo dos exames coprológicos.

Todas as crianças com resultados positivos foram tratadas com Tiabendazol para Strongyloides stercoralis e Mebendazol para os demais vermes, empregando-se o esquema de $100 \mathrm{mg}$ duas vezes ao dia, durante três dias. Apesar de não ser objeto do presente estudo, os indivíduos positivos para protozoários foram tratados como preconizado ${ }^{13}$.

O tratamento conduzido, aliado a um Programa de Educação e Saúde em Verminose*, constituíram-se instrumentos de uma proposta de intervenção em helmintose, nessa população, nos anos de 1985 e 1986.

As condiçס̃es econômicas da amostra foram analisadas tomando-se como referência a variável "salário mínimo per capita" (SMPC), definido como o quociente do número de salários mínimos percebidos pelo número de pessoas que compunham a família. Foram estudadas as associaçס̃es entre helmintoses e o tamanho da família, anemia e desnutrição e SMPC. A técnica estatística utilizada foi a do qui-quadrado para testes de associação em tabelas de contingência, considerando-se o nível crítico de significância igual a $5 \%$. As análises estatísticas foram processadas em computador IBM 370/148 da Universidade Federal de São Carlos, usando-se programas do sistema BMDP (Biomedical Computer Programs).
Nem todas as crianças cadastradas participaram da coleta de sangue ou dos três levantamentos coprológicos. Vários fatores foram responsáveis por esse fato: a mudança de cidade, ausência na escola no período de corte de cana, dificuldade na coleta ou entrega do espécime fecal, entre outros.

\section{RESULTADOS E DISCUSSÃO}

Os resultados que traçaram o perfil sócioeconômico de Santa Eudóxia foram obtidos a partir de levantamento descritivo, o qual abrangeu 95 residências de famílias de escolares. Essas residências correspondiam a $51 \%$ dos domicílios existentes na vila entre os quais $96,7 \%$ eram de alvenaria e $93,6 \%$ possuiam mais de quatro cômodos.

Todas essas residências possuíam fossa e eram abastecidas pela rede pública de água, $73,1 \%$ das famílias eram proprietárias do imóvel onde residiam.

No levantamento sobre relações de trabalho verificou-se que $55,9 \%$ dos chefes de família eram proprietários de terra ou de estabelecimentos comerciais, e $44,1 \%$ eram lavradores trabalhando no cultivo da cana, café e laranja.

As 254 crianças estudadas forneceram um total de 643 amostras fecais nos três levantamentos, sendo 217,252 e 174 em cada semestre, respectivamente, como mostra a Tabela 1 .

O total de resultados positivos foi registrado como segue: $82(37,8 \%)$ na primeira coleta, 109 $(43,2 \%)$ na segunda e $37(21,3 \%)$ na terceira. Observa-se que a espécie de helminto dominan. te no total geral foi $A$. lumbricoides $(12,1 \%)$, seguido de T.trichiura $(4,8 \%)$, ancilostomídeo $(3,7 \%)$ e $H$. nana $(3,7 \%)$. A baixa positividade de Taenia sp., S. stercoralis e E. vermicularis deve ser vista com cautela, uma vez que o método empregado não foi específico para esses parasitas. As associaçס̃es de duas ou mais espécies em cada coleta ocorreram em baixa percentagem. As mais frequientes foram representadas por Ascaris + Trichuris $(2,8 \%)$, Ascaris + ancilostomídeo $(1,9 \%)$ e ancilostomídeo + Trichuris $(1,2 \%)$, como era esperado, uma vez que essas três espécies foram isoladamente mais prevalentes.

A Tabela 1 revela também que os helmintos $A$. lumbricoides, ancilostomídeo e $T$. trichiura mostraram-se sensíveis às medidas profiláticas aplicadas durante o estudo, reduzindo suas freqüências iniciais; por outro lado, $H$. nana teve

* Dados inéditos, devendo ser objeto de próxima publicação. 
TABELA 1

Prevalência de helmintos intestinais em escolares, Subdistrito de Santa Eudóxia, Município de São Carlos, Estado de São Paulo, 1985:1986

\begin{tabular}{|c|c|c|c|c|c|c|c|c|}
\hline \multirow{3}{*}{ Espécies de Helmintos } & \multicolumn{6}{|c|}{ Prevalênicia } & \multirow{3}{*}{$\begin{array}{l}\text { Total } \\
\text { Geral }\end{array}$} & \multirow[t]{3}{*}{$\%$} \\
\hline & \multicolumn{2}{|c|}{19 Sem. 1985} & \multicolumn{2}{|c|}{19 Sem. 1986} & \multicolumn{2}{|c|}{ 20. Sem. 1986} & & \\
\hline & $\mathrm{T}$ & $\%$ & $\mathrm{~T}$ & $\%$ & $\mathrm{~T}$ & $\%$ & & \\
\hline \multirow{12}{*}{$\begin{array}{l}\text { Ascaris lumbricoides } \\
\text { Ancilostomídeo } \\
\text { Trichuris trichiura } \\
\text { Hymenolepis nana } \\
\text { Taenia sp } \\
\text { Strongyloides sterco- } \\
\text { ralis } \\
\text { Enterobius vermicula. } \\
\text { ris } \\
\text { Ascaris + Ancilostomí- } \\
\text { deo } \\
\text { Ascaris + Trichuris } \\
\text { Ancilostomídeo +Tri- } \\
\text { churis } \\
\text { Outras Associaçōes Du- } \\
\text { plas } \\
\text { Outras Associaçס̄es Tri- } \\
\text { plas }\end{array}$} & 36 & 16,6 & 31 & 12,3 & 11 & 6,3 & 78 & 12,1 \\
\hline & 12 & 5,5 & 8 & 3,2 & 4 & 2,3 & 24 & 3,7 \\
\hline & 10 & 4,6 & 16 & 6,3 & 5 & 2,8 & 31 & 4,8 \\
\hline & 6 & 2,8 & 7 & 2,8 & 11 & 6,3 & 24 & 3,7 \\
\hline & 2 & 0,9 & - & - & - & - & 2 & 0,3 \\
\hline & - & - & 4 & 1,6 & 1 & 0,6 & 5 & 0,8 \\
\hline & - & - & 2 & 0,8 & - & - & 2 & 0,3 \\
\hline & 8 & 3,7 & 4 & 1,6 & - & - & 12 & 1,9 \\
\hline & 4 & 1,8 & 12 & 4,7 & 2 & 1,2 & 18 & 2,8 \\
\hline & 1 & 0,5 & 5 & 2,0 & 2 & 1,2 & 8 & 1,2 \\
\hline & 2 & 0,9 & 13 & 5,1 & - & - & 15 & 2,3 \\
\hline & 1 & 0,5 & 7 & 2,8 & 1 & 0,6 & 9 & 1,4 \\
\hline Total Positivos & 82 & 37,8 & 109 & 43,2 & 37 & 21,3 & 228 & 35,3 \\
\hline Total Negativos & 135 & 62,2 & 143 & 56,7 & 137 & 78,7 & 415 & 64,5 \\
\hline $\begin{array}{l}\text { Total Amostras Exa- } \\
\text { minadas }\end{array}$ & 217 & 100,0 & 252 & 100,0 & 174 & 100,0 & 643 & 100,0 \\
\hline
\end{tabular}

TABELA 2

Distribuição dos exames positivos por helmintos intestinais e faixa etária, Subdistrito de Santa Eudóxia, Município de São Carlos, Estado de São Paulo, 1985-1986

\begin{tabular}{cccccccc}
\hline & \multicolumn{7}{c}{ Exame de Fezes } \\
\cline { 2 - 6 } Faixa Etária & \multicolumn{2}{c}{ Negativos } & \multicolumn{2}{c}{ Positivos } & & Total & $\%$ \\
\cline { 2 - 6 } & No & $\%$ & No & $\%$ & & \\
\hline $3-1$ & 39 & 29,8 & 23 & 29,1 & 62 & 29,5 \\
812 & 60 & 45,8 & 41 & 51,9 & 101 & 48,1 \\
$\geqslant 12$ & 32 & 24,4 & 15 & 19,0 & 47 & 22,4 \\
\hline Total & 131 & 100,0 & 79 & 100,0 & 210 & 100,0 \\
\hline
\end{tabular}

$X_{2}^{2}=1,04 \quad P=0,59$

sua prevalência aumentada. Os percentuais de positividade para esta última espécie, por levantamento, foi de $2,8 \%, 2,8 \%$ e $6,3 \%$, respectivamente. Observaçðes semelhantes foram feitas por Nussenzveig e col. ${ }^{9}$, em escolares do Município de São Paulo. Sugerem esses autores que a persistência de $H$. nana após a intervenção se deve a insucessos terapêticos, visto que para esse helminto a dose de Mebendazol preconizada é o dobro daquela empregada para outros parasitas e, além disso, a Niclosamida e o Pra- ziquantel são as drogas de eleição no tratamento da hemenolepiase.

A distribuição dos resultados do primeiro exame de fezes por faixa etária (antes da aplicação de medidas de intervenção) encontra-se na Tabela 2 e revela que o estrato de 8 a 12 anos apresentou a maior positividade $(51,9 \%)$, enquanto aquele com crianças acima de 12 anos mostrou a frequiência mais baixa. Todavia essas diferenças não são estatisticamente significantes $\left(X^{2}=1,04 \% ; p=0,59 \%\right)$, sugerindo que em 
nossa amostra não há efeito de estratificação etária sobre a distribuição das helmintoses.

Os resultados da análise de associação entre o tamanho da familia e a distribuição dos resulta. dos de exames coprológicos estão na Tabela 3. A análise estatística mostrou relação significante entre as duas variáveis acima mencionadas $\left(X^{2}=10,00 ; p=0,007\right)$; verifica-se que enquanto nas famílias com oito ou mais pessoas os resultados positivos chegam a $29,2 \%$, naquelas com quatro ou menos pessoas foi observada uma baixa positividade $(6,1 \%)$. Algumas hipóteses foram levantadas na tentativa de explicar esse resultado: a) nessa amostra a variável "tamanho familiar" é um índice da condição sócio-econômica bastante sensível para detectar os efeitos da distribuição das helmintoses; b) ocorre de fato uma agregação familiar na distribuição das helmintoses; c) ao se rejeitar a hipótese nula, isto é, de não associação entre essas variáveis, foi cometido um erro estatístico do tipo I (rejeitar a hipótese nula quando ela é verdadeira).

Um teste indireto da primeira hipótese está na Tabela 4 , onde se analisa a relação entre a variável SMPC e helmintos intestinal, e se verifica que não houve associação estatisticamente significante entre elas $\left(X^{2}=0,46 ; 0,8<p<0,9\right)$. Como já foi mencionado, SMPC é um índice sócio-econômico em cuja conceituação figura a variável "tamanho familiar"; o fato de não se detectar correlação significante entre SMPC e helmintose, sugere uma falta de consistência na relação entre tamanho familiar e resultados dos exames coprológicos.

Quanto a segunda hipótese, há na literatura estudo ${ }^{11}$ que sugere a agregação familiar como uma variável que interfere na distribuição das helmintoses. Contudo, seu teste torna-se impossivel no presente estudo, visto que a amostra atual não inclui resultados dos exames coprológicos da família dos escolares averiguados. Assim, a atitude mais cautelosa é a admissão de um erro do tipo I para explicar a associação encontrada.

Em relação à análise da anemia, verificou-se que a taxa média de hemoglobina foi de $13,06 \pm 1,00 \mathrm{gr} / \mathrm{dl}$. De acordo com o critério da OMS, em $16(9,3 \%)$ crianças entre"as 172 examinadas foi constatada anemia. A Tabela 5

TABELA 3

Correlação entre composição familiar e resultados dos exames coprológicos, Subdistrito de Santa Eudóxia, Município de São Carlos, Estado de São Paulo, 1985-1986

\begin{tabular}{|c|c|c|c|c|c|c|}
\hline \multicolumn{7}{|c|}{ Resultados dos Exames Coprológicos } \\
\hline \multirow{2}{*}{$\begin{array}{l}\text { Composição } \\
\text { Familiar }\end{array}$} & \multicolumn{2}{|c|}{ Negativo } & \multicolumn{2}{|c|}{ Positivo } & \multicolumn{2}{|c|}{ Total Geral } \\
\hline & no & $\%$ & n! & $\%$ & no & $\%$ \\
\hline $\begin{array}{c}\leqslant 4 \\
5-7 \\
\geqslant 8\end{array}$ & $\begin{array}{l}26 \\
61 \\
20\end{array}$ & $\begin{array}{l}24,3 \\
57,0 \\
18,7\end{array}$ & $\begin{array}{r}4 \\
42 \\
19\end{array}$ & $\begin{array}{r}6,1 \\
64,6 \\
29,2\end{array}$ & $\begin{array}{r}30 \\
103 \\
39\end{array}$ & $\begin{array}{l}17,4 \\
59,9 \\
22,7\end{array}$ \\
\hline Total & 107 & 100,0 & 65 & 100,0 & 172 & 100,0 \\
\hline
\end{tabular}

$X_{2}^{2}=10,00 \quad P=0,007$

TABELA 4

Distribuição dos resultados dos exames de fezes por salário mínimo per capita (SMPC) no Subdistrito de Santa Eudóxia, Município de São Carlos, Estado de São Paulo, 1986

\begin{tabular}{|c|c|c|c|c|c|c|}
\hline \multirow{3}{*}{ SMPC } & \multicolumn{5}{|c|}{ Exame de Fezes } & \\
\hline & \multicolumn{2}{|c|}{ Negativo } & \multicolumn{2}{|c|}{ Positivo } & \multicolumn{2}{|c|}{ Total } \\
\hline & No & $\%$ & Nọ & $\%$ & Nọ & $\%$ \\
\hline $\begin{array}{l}<1 \\
\geqslant 1\end{array}$ & $\begin{array}{l}88 \\
19\end{array}$ & $\begin{array}{l}82,2 \\
17,7\end{array}$ & $\begin{array}{r}56 \\
9\end{array}$ & $\begin{array}{l}86,1 \\
13,8\end{array}$ & $\begin{array}{r}144 \\
28\end{array}$ & $\begin{array}{l}83,7 \\
16,3\end{array}$ \\
\hline Total & 107 & 100,0 & 65 & 100,0 & 172 & 100,0 \\
\hline
\end{tabular}

$\mathrm{X}_{1}^{2}=0,46 \quad 0,8<\mathrm{p}<0,9$ 
mostra que não é significante a associação entre anemia e distribuição dos resultados do exame de fezes $\left(X^{2}=0,7 ; p=0,4\right)$. Esse resultado vem reforçar as observações anteriores feitas por Jorge João e col. ${ }^{2}$, em crianças do Estado do Pará, e Sigulem e col. $12 \mathrm{em}$ amostra de crianças de São Paulo. Corroborando com os resultados desses autores, conclui-se que os dados de Santa Eudóxia evidenciam falta de associação entre verminose e anemia, pelo menos a nível populacional.

$\mathrm{Na}$ Tabela 6 estão as observações entre helmintoses e desnutrição. Embora a taxa de desnutrição em nossa amostra seja de magnitude considerável $(14,4 \%)$, a análise dos dados mostra que não houve significância estatística nessa correlação $\left(\mathrm{X}^{2}=0,36 ; 0,7<\mathrm{p}<0,8\right)$. Portanto, na situação estudada as helmintoses detectadas não são causa de desnutrição em nossa população.

O estudo de coorte compreendeu 94 crianças $(43,3 \%$ da primeira amostra) que foram acompanhadas para a observação da dinâmica da infecção parasitária; de 38 resultados positivos, na primeira amostra, $28(73,7 \%)$ mostraram-se negativos no último exame, após o período de intervenção: das 56 crianças que mostraram resultados negativos na primeira coleta, 50 $(89,3 \%)$ continuaram negativos no último levantamento. Esses resultados sugerem que as medidas de intervenção (tratamento e educação) utilizadas em Santa Eudóxia possam ter sido responsáveis em parte pela evolução da negativação de helmintos nos exames de fezes.

Durante o período no qual o trabalho foi conduzido, várias modificações surgiram em Santa Eudóxia como, por exemplo, o asfaltamento da estrada vicinal, construção da creche e de um novo posto de saúde (ambos em funcionamento). A resultante destas modificaçōes, melhoria das condiç̃es da população, influiu decisiva $e$ inversamente na prevalência das helmintoses nessa vila. Conclui-se deste fato, acrescido ao curto período de observação para um projeto de intervenção, que se faz necessário uma avaliação, dentro de um período maior, da eficácia dos métodos de intervenção utilizados, os quais a curto prazo mostraram-se eficazes.

TABELA 5

Distribuição da taxa de anemia e resultados de exames parasitológicos, Subđistrito de Santa Eudóxia, Município đe São Carlos, Estado de São Paulo, 1985-1986

\begin{tabular}{|c|c|c|c|c|c|c|c|}
\hline \multirow[b]{2}{*}{ Anemia } & \multirow[t]{2}{*}{ Fezes } & \multicolumn{2}{|c|}{ Negativo } & \multicolumn{2}{|c|}{ Positivo } & \multicolumn{2}{|c|}{ Total } \\
\hline & & No & $\%$ & Nọ & $\%$ & No & $\%$ \\
\hline $\begin{array}{l}\text { Normal } \\
\text { Anêmico }\end{array}$ & & $\begin{array}{l}95 \\
12\end{array}$ & $\begin{array}{l}88,8 \\
11,2\end{array}$ & $\begin{array}{r}61 \\
4\end{array}$ & $\begin{array}{r}93,8 \\
6,1\end{array}$ & $\begin{array}{r}156 \\
16\end{array}$ & $\begin{array}{r}90,7 \\
9,3\end{array}$ \\
\hline Total & & 107 & 100,0 & 65 & 100,0 & 172 & 100,0 \\
\hline
\end{tabular}

$\mathrm{x}_{1}^{2}=0,7 \mathrm{p}=0,4$

TABELA 6

Distribuição de crianças segundo exames de fezes para helmintos e estado nutricional, Subdistrito de Santa Eudóxia, Município de São Carlos, Estado de São Paulo, 10985-1986

\begin{tabular}{|c|c|c|c|c|c|c|}
\hline \multirow{3}{*}{$\begin{array}{c}\text { Estado } \\
\text { Nutricional }\end{array}$} & \multicolumn{6}{|c|}{ Exames de Fezes } \\
\hline & \multicolumn{2}{|c|}{ Negativo } & \multicolumn{2}{|c|}{ Positivo } & \multicolumn{2}{|c|}{ Total } \\
\hline & Nọ & $\%$ & No & $\%$ & Nọ & $\%$ \\
\hline $\begin{array}{l}\text { Desnutrido } \\
\text { Não Desnutrido }\end{array}$ & $\begin{array}{r}17 \\
111\end{array}$ & $\begin{array}{l}13,3 \\
86,7\end{array}$ & $\begin{array}{l}12 \\
61\end{array}$ & $\begin{array}{l}16,4 \\
83,5\end{array}$ & $\begin{array}{r}29 \\
172\end{array}$ & $\begin{array}{l}14.4 \\
85.6\end{array}$ \\
\hline Total & 128 & 100,0 & 73 & 100,0 & 201 & 100,0 \\
\hline
\end{tabular}

$x_{1}^{2}=0,36 \quad 0,7<p<0,8$ 


\section{AGRADECIMENTOS}

À Direção do Instituto Adolfo Lutz, do Município de São Carlos, pela execução dos exames laboratoriais; ao Prof. Mauro Fisberg, da
Escola Paulista de Medicina, pelas sugestoes à primeira versão deste trabalho, e às estagiárias Gláucia E.G. Ferreira, Solange C.G. Ribeiro e Regina Abraão Dias, pela presteza na coleta do material e organização dos dados.

PEDRAZZANI, E.S. et al. [Intestinal helminthiasis. II - Prevalence, correlation with income, family size, anemia and nutritional status. Rev. Saúde públ., S. Paulo, 22:384-9, 1988.

ABSTRACT: This survey analises the prevalence of the intestinal helminthiasis and their correlation with anemia, nutritional status, income and family size in school children in Santa Eudoxia (S. Carlos, SP, Brazil). Results achieved by the Lutz method performed on fecal samples from a total of 254 children showed a prevalence of $12.1 \%$ for Ascaris lumbricoides, $3.7 \%$ for ancylostomideo, $4.8 \%$ for Trichuris trichiura and $3.7 \%$ for Hymenolepis nana. The highest level of infestation (51.9\%) occurred in the 8 to 12-year age groups. An incidence of $9.3 \%$ of anemia and $14.4 \%$ of malnutrition was detected. Only one of the associations studied was statistically significant - that between helminthiasis and family size.

UNITERMS: Helminthiasis, occurrence. Health surveys. Anemia, occurrence. Nutritional status. Income. Family characteristics. Feces, analysis.

\section{REFERÊNCIAS BIBLIOGRÁFICAS}

1. BLOCK, M. El parasitismo intestinal un tabu que debe ser destruido. Rev. Inst. Invest. Med., 10(2):102-7, 1981.

2. JORGE JOÃO, W.S. et al. Prevalência de desnutrição em crianças menores de 6 anos de idade atendidas no serviço ambulatorial da Santa Casa do Pará. Hiléia med., Belém, 4(2):33-42, 1983.

3. LOTERO, H. et al. Gastrointestinal blood loss in Trichuris infection. Amer. J. trop. Med. Hyg., 23:12034, 1974.

4. MARQUES, R.M. et al. Crescimento e desenvolvimento pubertário em crianças e adolescentes brasileiros. II. Altura e peso. São Paulo, Editora Brasileira de Ciências, 1982.

5. MASPES, V. et al. Importância da reabsorção do ferro da hemorragia intestinal provocada pela ação dos vermes na progressão da anemia. Rev. Saúde públ., S. Paulo, 18:209-17, 1984.

6. MELLO, D.A. et al. Helmintoses intestinais no Subdistrito de Santa Eudóxia (Município de São Carlos SP). I - Conhecimentos, atitudes e percepção da população sobre helmintos e helmintoses intestinais. Rev. Saúde públ., S. Paulo, 22:140-9, 1988.

7. MIALE, J.B. Laboratory medicine hematology. St. Louis, C.V. Mosby, 1970.
8. MONTEIRO, C.A. O problema da desnutrição no Estado de São Paulo (Brasil): informaçōes disponíveis, lacunas no conhecimento e linhas de pesquisa prioritárias. Rev. Saúde públ., S. Paulo, 19:171-82, 1985.

9. NUSSENZVEIG, I. et al. Prevalência de anemia e de parasitoses intestinais em escolares do municipio de São Paulo: resultados do emprego da merenda escolar e das drogas antiparasitárias. Rev. paul. Med., 100:32-9, 1982.

10. ORGANIZACION MUNDIAL DE LA SALUD. Grupo de Expertos en Anemias Nutricionales, Ginebra, 1971. Informe. Ginebra, 1972. (Série de Informes Técnicos, 503).

11. ROSABAL, R. \& LUNA, D. Parasitismo familiar. Rev. Centromer. Cienc. Salud, 6(1):9-17, 1977.

12. SIGULEM, D.M. et al. Anemia nutricional e parasitose intestinal em menores de 5 anos. Rev. paul. Med., 103:308-12, 1985.

13. VERONESI, R. Doenças infecciosas e parasitárias. São Paulo, Ed. Guanabara Koogan, 1972.

Recebido para publicação em 18/11/1987 Reapresentado em 4/8/1988 Aprovado para publicação em 5/8/1988 